\title{
Identification of PSEN1 and PSEN2 Gene Variants and Clinical Findings with the Literature
}

\author{
Nadide Cemre Randa ${ }^{1 *}$, Elçin Bora ${ }^{1}$, Esra Ataman ${ }^{1}$, Özlem Öz ${ }^{1}$, Görsev Yener ${ }^{2}$ and Ayfer Ülgenalp ${ }^{1}$
}

${ }^{1}$ Department of Medical Genetics, Dokuz Eylul University Medicine Faculty, Turkey

${ }^{2}$ Department of Neurology, Dokuz Eylul University Medicine Faculty, Turkey

*Corresponding author: Nadide Cemre Randa, Department of Medical Genetics, Dokuz Eylul University Medicine Faculty, Turkey, Tel: +90536-778-38-37

\section{Introduction}

Alzheimer's disease is an age-related and irreversible disease and characterized progressively decline in cognitive functions such as memory, speech, executive functions and visual-spatial skills. Currently, there is no a simple and definitive diagnosis method for clinical management [1]. It is possible to diagnose with $85-90 \%$ accuracy with clinical assesment and neuropsychological tests [2]. The precisely-known risk factors associated with Alzheimer's disease are aging, family history, and the presence of the Apolipoprotein E \&4 (APO E4) allele [3]. Alzheimer's disease is usually sporadic and about only $1-5 \%$ is familial. Amyloid precursor protein (APP), Presenilin 1 (PSEN1), and Presenilin 2 (PSEN2) genes cause to familial early onset Alzheimer's disease and account for less than $1 \%$ of all Alzheimer's patients [1]. Missense mutations in PSEN1 gene are the most common cause of Alzheimer's disease and there are 219 pathogenic mutations reported in the literature. To date, there are 51 pathogenic mutations in APP and 16 pathogenic mutations reported in the literature [4].

\section{Materials and Methods}

In this study we analyzed 81 patients who referred to our outpatient clinic diagnosed as early onset Alzheimer's disease (EOAD) between January 2014-January 2017 and had been performed Sanger sequencing analysis for exons 16 and 17 of APP, and all coding exons of PSEN1 and PSEN2 genes. All sequenced patients were demented, and of the 81 patients, 49 were female and 32 were male. The average age at admission and mean age of onset were 54.8 (Patients were referred to our outpatient clinic at the time of diagnosis). Before testing, written informed consent was obtained from the patients and/or family members, and ethics committee approval was received for this study from the Ethics Committee of Dokuz Eylul University Medicine Faculty. We found seven PSEN1and four PSEN2 mutations, and we did not find any APP mutations. To analyze effects of PSEN1 and PSEN2 mutations on age onset, we performed Kruskal Wallis test and there is no significant difference between these genes ( $p: 0.06)$. Then we performed Mann Whitney $U$ test to analyze age onset differences between patient groups that bearing PSEN1 or PSEN2 mutations and the second group that bearing any mutations in APP, PSEN1 and PSEN2 genes. Mann Whitney $U$ test showed significant difference between the first patient group that bearing PSEN1 or PSEN2 mutations and the second group that bearing any mutations in APP, PSEN1 and PSEN2 genes ( $p: 0.038$ ). This result means age of onset is younger in patients that bearing PSEN1 and PSEN2 mutations as expected.

\section{Statistical analysis}

Statistical data processing was performed using Statistical Package for the Social Sciences program (SPSS 15.0; IBM) to assess the significance of differences in the results using Kruskal Wallis and Mann Whitney $U$ tests. Statistical significance level of $p<0.05$ was accepted.

\section{Results}

When we performed Sanger sequencing, we detected seven patients with PSEN1 gene mutations and four patients with PSEN2 gene mutations within 81 patients.

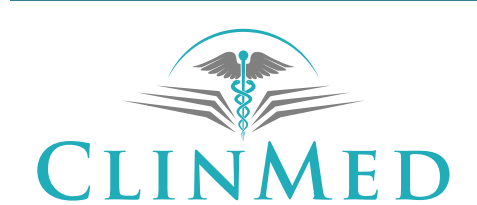

INTERNATIONAL LIBRARY
Citation: Randa NC, Bora E, Ataman E, Öz O, Yener G, et al. (2019) Identification of PSEN1 and PSEN2 Gene Variants and Clinical Findings with the Literature. Int J Neurodegener Dis 2:007

Accepted: April 09, 2019; Published: April 11, 2019

Copyright: (c) 2019 Randa NC, et al. This is an open-access article distributed under the terms of the Creative Commons Attribution License, which permits unrestricted use, distribution, and reproduction in any medium, provided the original author and source are credited. 
Table 1: Detected mutation and their prediction of pathogenicity and clinical findings.

\begin{tabular}{|l|l|l|l|l|}
\hline Mutation & $\begin{array}{l}\text { Prediction of } \\
\text { pathogenicity }\end{array}$ & $\begin{array}{l}\text { Age } \\
\text { onset }\end{array}$ & Initial symptom & $\begin{array}{l}\text { Previous publications and/or } \\
\text { molgen database }\end{array}$ \\
\hline PSEN1 p.H163R & Pathogenic variant & 43 & Unknown & Yes \\
\hline PSEN1 p.H163Y & Pathogenic variant & 54 & Memory deficit & Yes \\
\hline PSEN1 p.L173F & Pathogenic variant & 44 & Unknown & Yes \\
\hline PSEN1 p.V193G & Uncertain significance & 53 & $\begin{array}{l}\text { Obsessive behaviour, } \\
\text { paranoid thoughts }\end{array}$ & No (ExAc browser) \\
\hline PSEN1 p.I213F & Pathogenic variant & 34 & Memory deficit & Yes \\
\hline PSEN1 p.E280A & Pathogenic variant & 45 & Memory deficit & Yes \\
\hline PSEN1 p.A431V & Pathogenic variant & 61 & Unknown & Yes \\
\hline PSEN2 p.T430M & Pathogenic variant & 52 & Memory deficit & Yes \\
\hline PSEN2 c.142-7delC & Uncertain significance & 54 & Word finding difficulty & No (Novel variant) \\
\hline PSEN2 p.G34S & Uncertain significance & 55 & Memory deficit & Yes \\
\hline PSEN2 p.R62C & Uncertain significance & 54 & Unknown & Yes \\
\hline
\end{tabular}

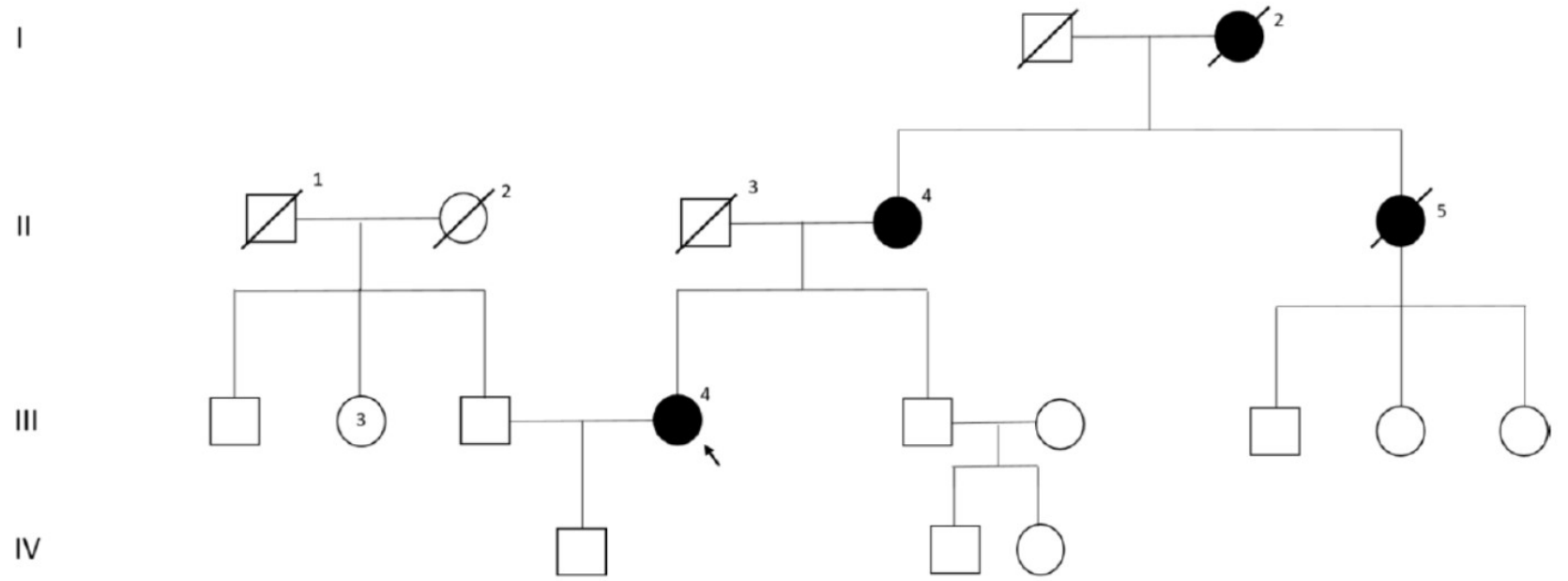

Early onset Alzheimer's disease

Figure 1: PSEN1 p.H163R mutated patient's pedigree (Arabic numerals represents the order of each individual in same generation row. Roman numerals, arrow, squares and circles represent the order of generations, proband, males and females, respectively).

11 mutated patients analyzed for disease onset symptoms and age of onset. We could reach the initial symptoms of five patients and three of them had memory deficits, one patient had word finding difficulties and one patient had psychiatric problems and our data is presented in Table 1.

\section{Patients}

The PSEN1 gene mutations in our group were His163Arg (H163R), His163Tyr (H163Y), Leu173Phe (L173F), Val193Gly (V193G), lle213Phe (I213F), Glu280Ala (E280A), and Ala431Val (A431V) mutations. The PSEN2 mutations were Thr430Met (T430M) and intronic c.1427delC mutations, and unknown significance variants were Gly34Ser (G34S) and Arg62Cys (R62C) variations.

\section{PSEN1 H163R}

Patient's symptoms onset at 43 years. As shown in Figure 1, patient's mother, aunt and grandmother had dementia and patient's mother had also H163R mutation. At the first visit, patient's Mini Mental Examination State (MMSE) score was 20, Blessed score was two and Instrumental Daily Living Activities (IDLA) score was 22. Brain Magnetic Resonance Imaging (MRI) shows cerebral atrophy.

\section{PSEN1 H163Y}

Patient's symptoms onset at 54 years. The initial symptom was memory loss and after two years patient was disoriented to space, repeated old stories over and over, and developed sleeping difficulties. As shown in Figure 2, the brother, mother and maternal aunt had early onset dementia and one of the maternal aunts had late onset dementia. Brain MRI shows diffuse atrophy.

\section{PSEN1 L173F}

Patient's disease onset age was 44 and brain MRI reveals cerebral atrophy. As shown in Figure 3 , the sister 


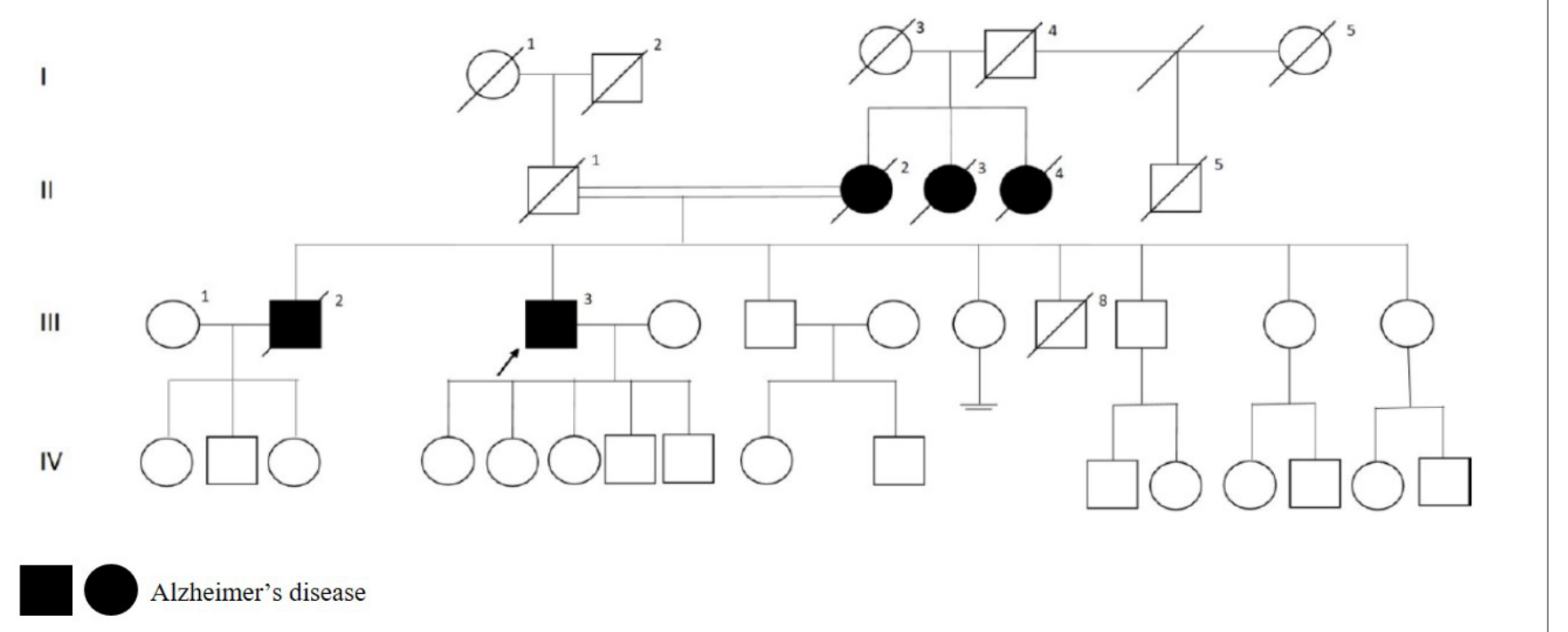

Figure 2: PSEN1 p.H163Y mutated patient's pedigree (Arabic numerals represents the order of each individual in same generation row. Roman numerals, arrow, squares and circles represent the order of generations, proband, males and females, respectively).

III

III
Forgetfulness and suicide attempt
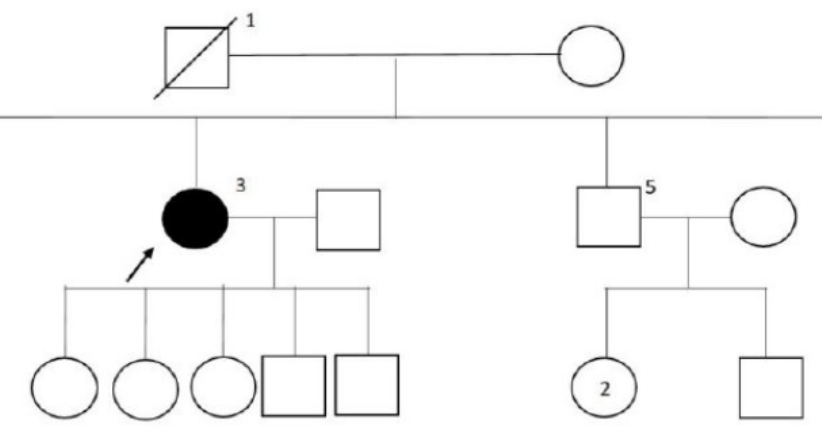
Alzheimer's disease
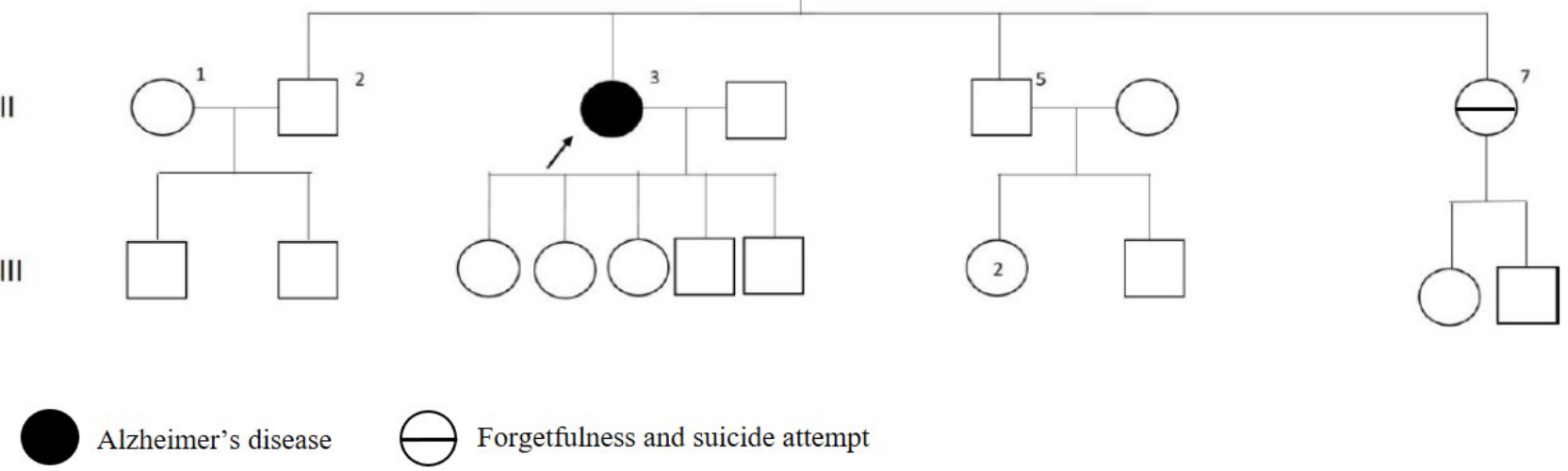

Figure 3: PSEN1 p.L173F mutated patient's pedigree (Arabic numerals represents the order of each individual in same generation row. Roman numerals, arrow, squares and circles represent the order of generations, proband, males and females, respectively).

had complained about memory loss and had suicide attempt.

\section{PSEN1 V193G}

The patient with V193G variation in PSEN1 gene had initial symptoms as obsessive compulsive disorder, paranoid thoughts and parkinsonism at when he was 53. MMSE score was 23 at the first visit and 14 after six months. Brain MRI was reported as biparietal atrophy. As shown in Figure 4, the father had early onset dementia, psychiatric problems and commited suicide and maternal aunt had late onset Alzheimer's disease.

\section{PSEN1 I213F}

The patient with I213F mutation in PSEN1 gene had initial symptoms as memory loss, sleep disturbances and personality changes at age 34 . After one and half years from the disease onset, word finding difficulty, parkinsonism and resting tremor on the right side were added to the clinical findings. As shown in Figure 5, there was no dementia in the family history. Brain MRI showed cerebral atrophy. MMSE was 23 at first visit and seven after six months.

\section{PSEN1 E280A}

Patient had an initial symptom as forgetfulness at age 45. MMSE was score 13 at the first visit and brain MRI was reported as cerebral atrophy. There was an early-onset dementia diagnosis of the patient's mother and 4 maternal aunts, and none were alive. As shown in Figure 6, there was a maternal uncle with dementia and panic attacks but the age of onset of the disease was unknown.

\section{PSEN1 A431V}

The patient with A431V mutation in PSEN1 gene had initial symptoms at age 61 and brain MRI was reported 


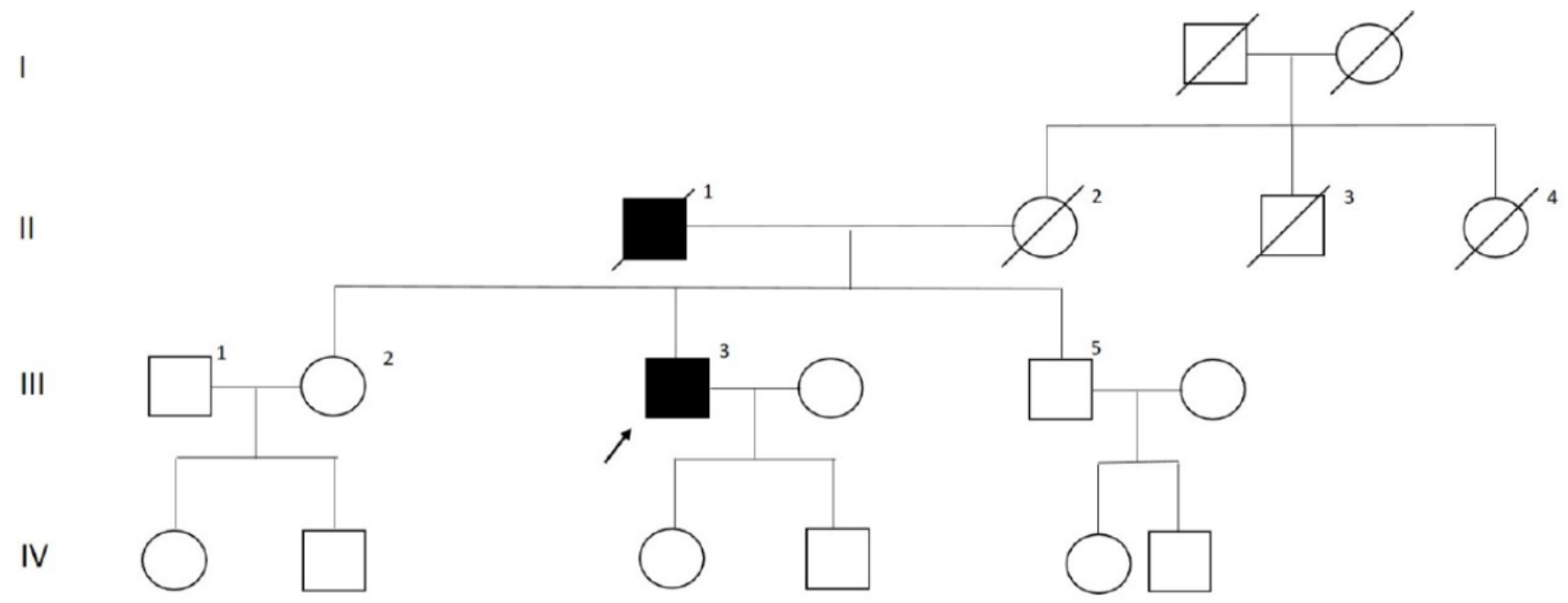

Early onset Alzheimer's disease and psychiatric symptoms

Figure 4: PSEN1 p.V193G mutated patient's pedigree (Arabic numerals represents the order of each individual in same generation row. Roman numerals, arrow, squares and circles represent the order of generations, proband, males and females, respectively).

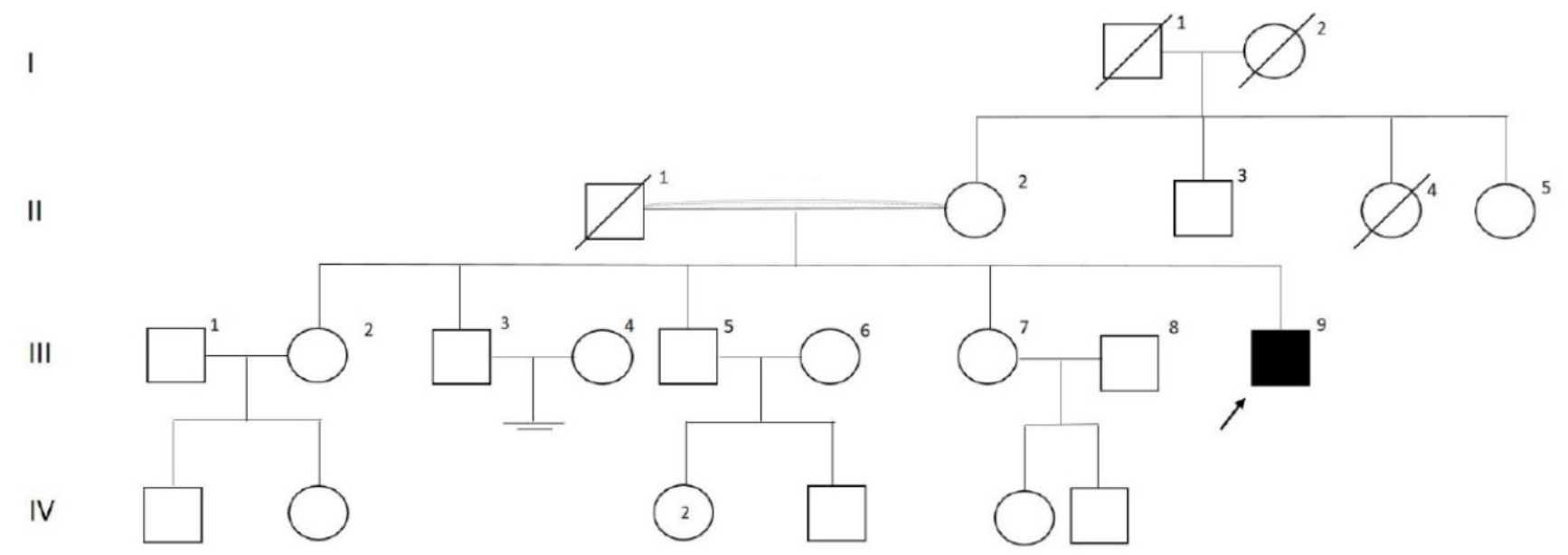

Early onset Alzheimer's disease

Figure 5: PSEN1 p.I213F mutated patient's pedigree (Arabic numerals represents the order of each individual in same generation row. Roman numerals, arrow, squares and circles represent the order of generations, proband, males and females, respectively).

as cerebral atrophy. As shown in Figure 7, the brother and father had early onset dementia and the mother had delirium due to medication.

\section{PSEN2 G34S}

The patient with G34S variation in PSEN2 gene had initial symptoms as mild memory loss, depression and lost in gained skills at age 55. After four years from the onset of the symptoms, the patient began to mix the rooms. MMSE score was two after 18 months from the first visit. Brain MRI was reported as chronic atrophy. As shown in Figure 8, the patient had no family history of dementia.

\section{PSEN2 c.142-7delC}

The patient with c.142-7delC variation in PSEN2 gene had initial symptom as word finding difficulty at age 54 . Two years after the onset of the symptoms reduced speech, introversion and forgetfulness developed. As shown in Figure 9, the patient had family history with late-onset Alzheimer's disease. The brain MRI was reported as cerebral and cerebellar atrophy.

\section{PSEN2 R62C}


I

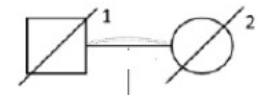

II

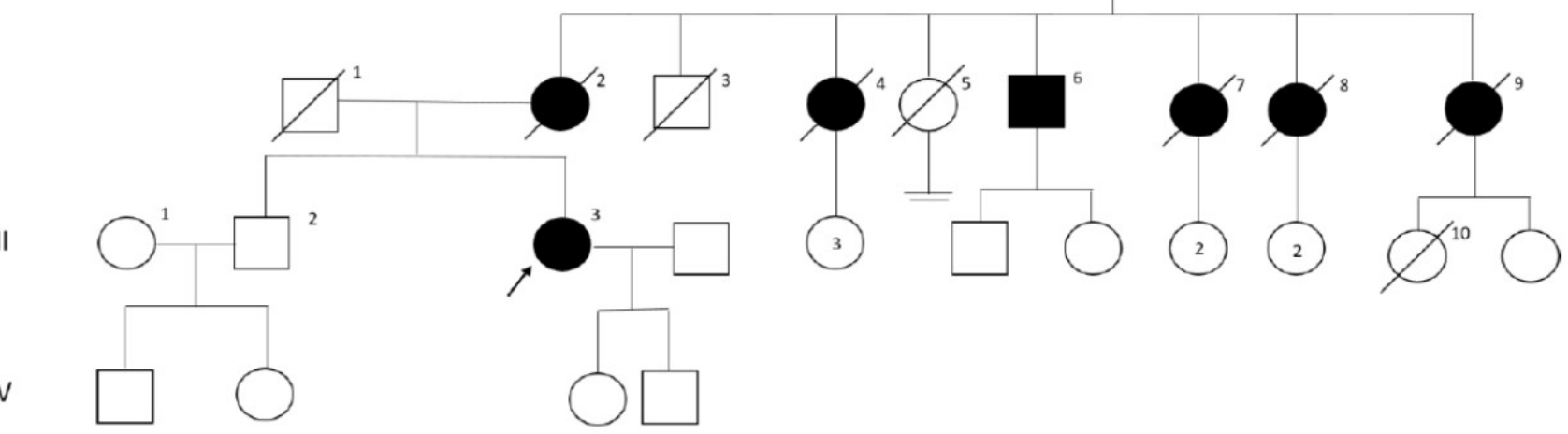

IV

Early onset dementia

Figure 6: PSEN1 p.E280A mutated patient's pedigree (Arabic numerals represents the order of each individual in same generation row. Roman numerals, arrow, squares and circles represent the order of generations, proband, males and females, respectively).

।

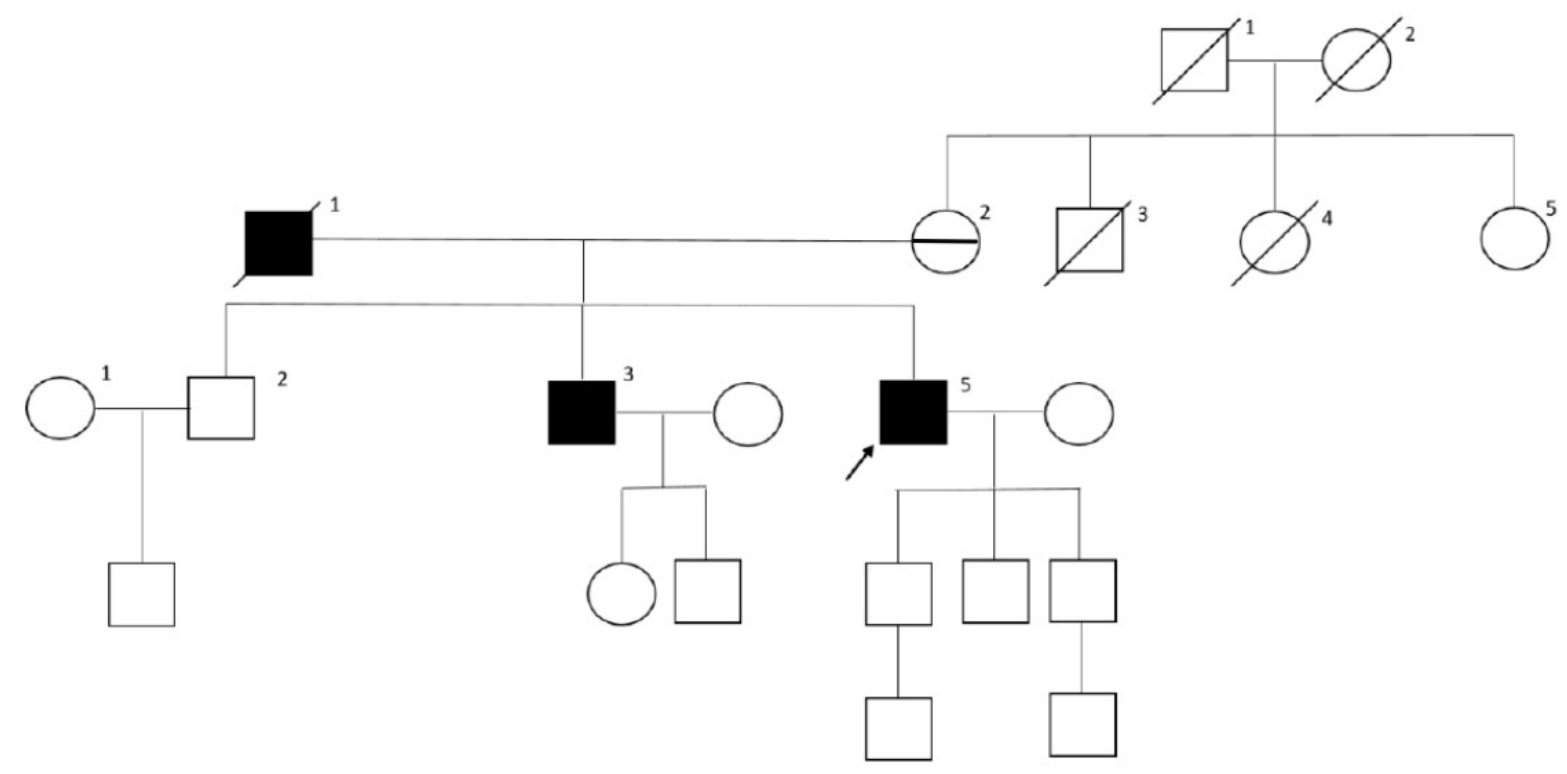

V

Early onset Alzheimer's disease

Delirium due to medication

Figure 7: PSEN1 p.A431V mutated patient's pedigree (Arabic numerals represents the order of each individual in same generation row. Roman numerals, arrow, squares and circles represent the order of generations, proband, males and females, respectively).

Patient had initial symptoms at age 54 . Brain MRI was normal. Her medical history included breast cancer and squamous cell carcinoma. As shown in Figure 10, the sister had suicide attempt and the mother had late onset dementia (diagnosis age at 91).

\section{PSEN2 T430M}

The patient with T430M variant in PSEN2 gene had initial symptoms as mild memory deficits and sleeping disturbances at age 52. As shown in Figure 11, the patient's mother had the same mutation. However, she was not demented when she was 78-year-old, only a general dissatisfaction was reported. Apart from this finding, maternal uncle who diagnosed as Alzheimer's after 70 years but could not be analyzed because he was not alive.

\section{Discussion}

Alzheimer's disease is a neurodegenerative and progressive disorder and still incurable. Autosomal dominant early-onset Alzheimer's disease constitutes 5-10\% of early-onset Alzheimer's disease and approximately 
II

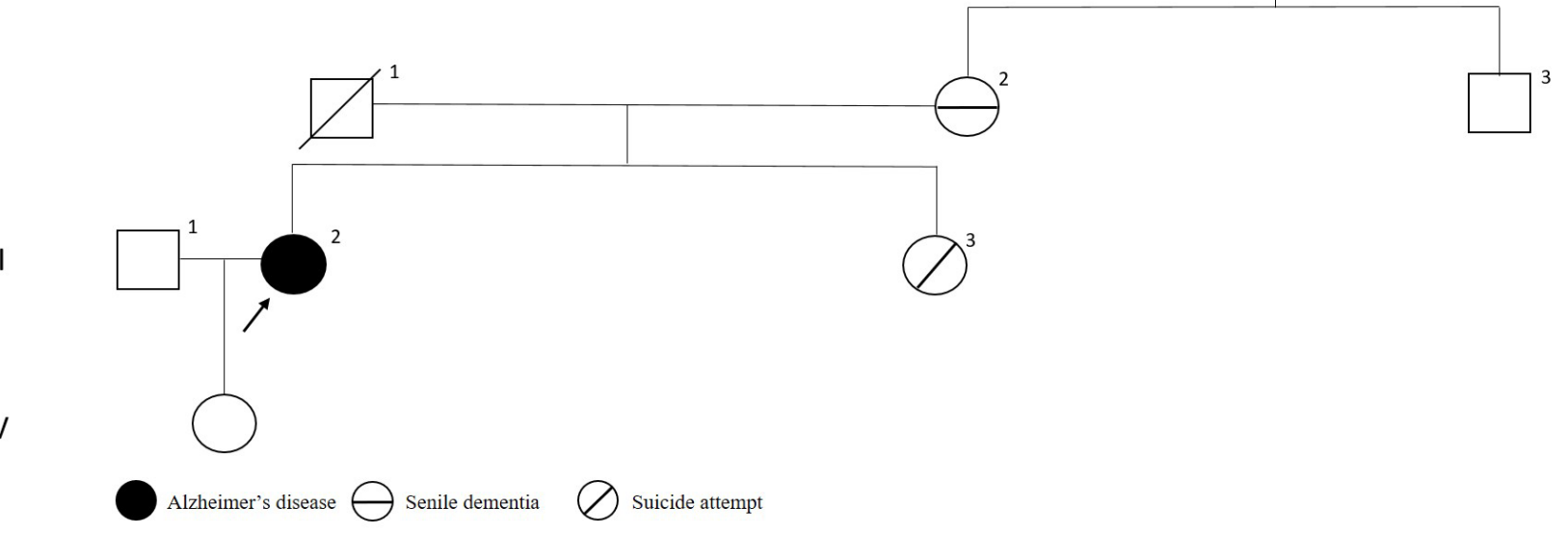

Figure 8: PSEN2 p.G34S mutated patient's pedigree (Arabic numerals represents the order of each individual in same generation row. Roman numerals, arrow, squares and circles represent the order of generations, proband, males and females, respectively).

I

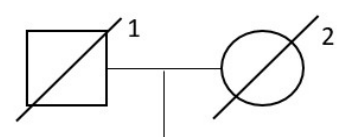

II

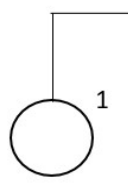

III

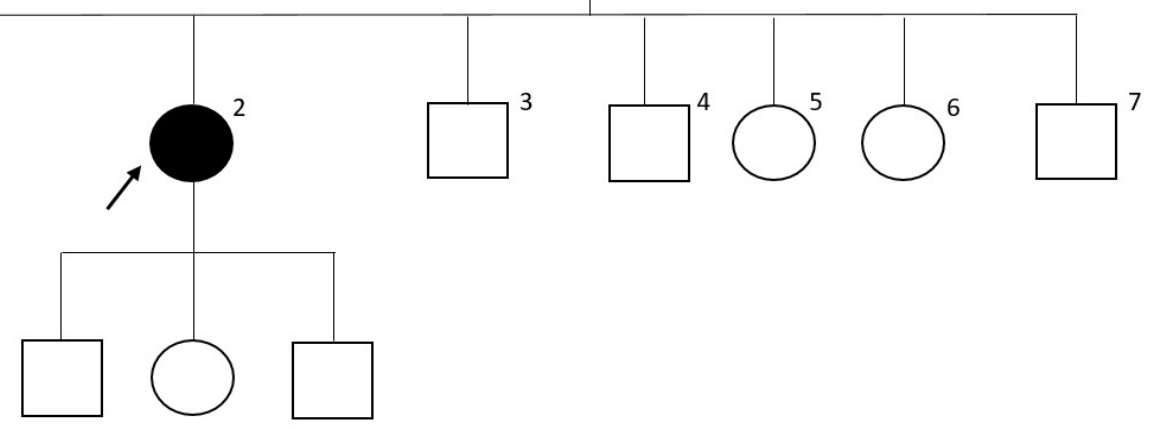

Early onset Alzheimer's disease

Figure 9: PSEN2 c.142-7delC mutated patient's pedigree (Arabic numerals represents the order of each individual in same generation row. Roman numerals, arrow, squares and circles represent the order of generations, proband, males and females, respectively).

I

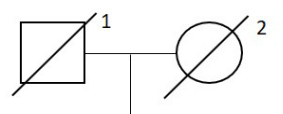

II

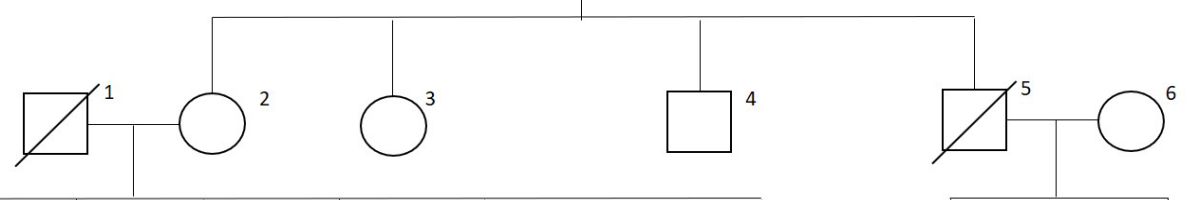

III
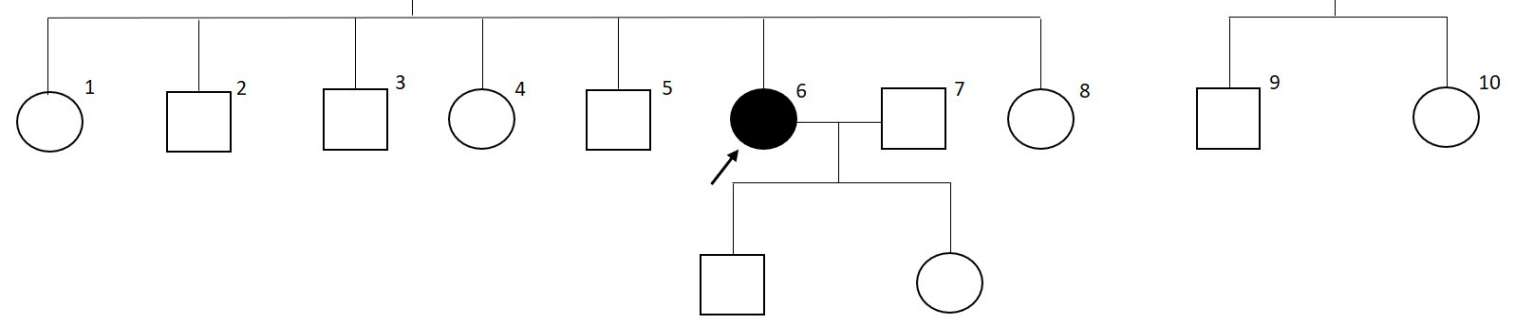

IV

Early onset Alzheimer's disease $\square$ Late onset Alzheimer's disease

Figure 10: PSEN2 p.R62C mutated patient's pedigree (Arabic numerals represents the order of each individual in same generation row. Roman numerals, arrow, squares and circles represent the order of generations, proband, males and females, respectively). 


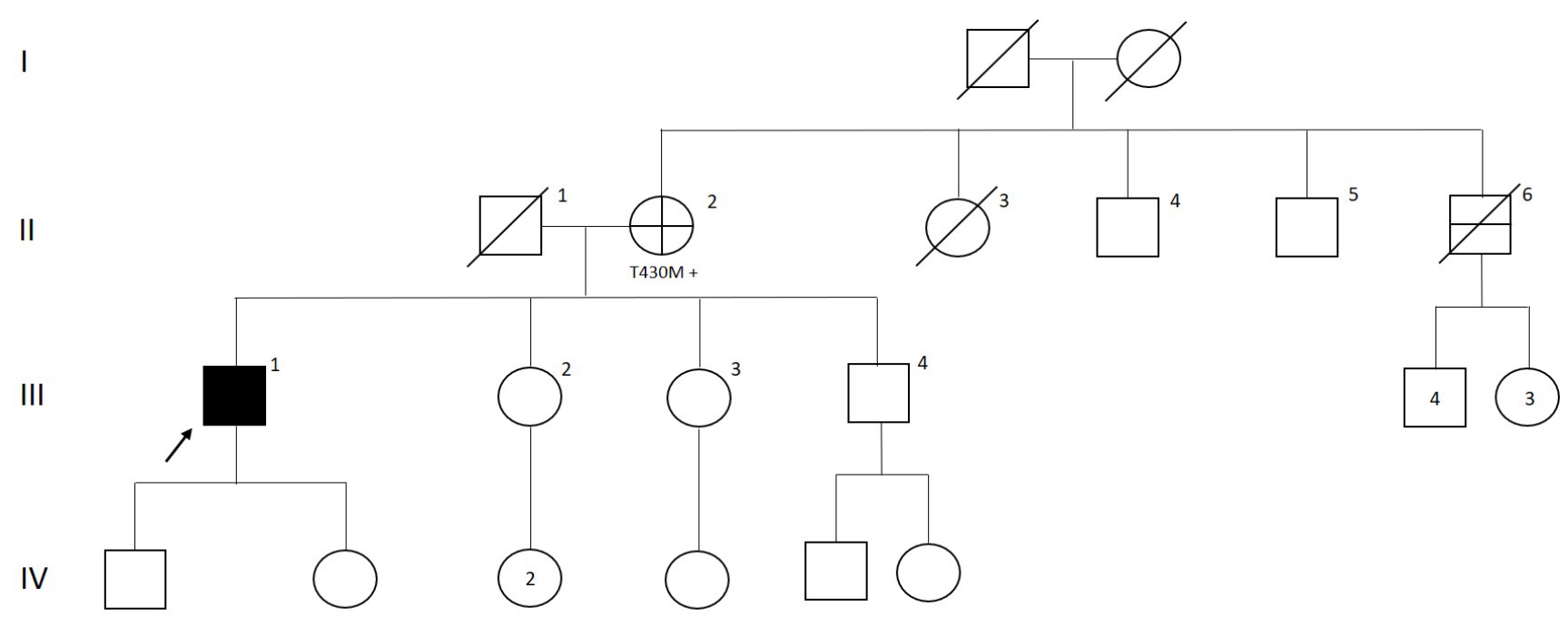

Early onset Alzheimer's disease

78 years-old, not demented, $\mathrm{T} 430 \mathrm{M}+$

Late onset Alzheimer's disease

Figure 11: PSEN2 p.T430M mutated patient's pedigree (Arabic numerals represents the order of each individual in same generation row. Roman numerals, arrow, squares and circles represent the order of generations, proband, males and females, respectively).

$1 \%$ of all Alzheimer patients [1]. APP mutations 10-15\%, PSEN1 mutations $30-70 \%$ and PSEN2 mutations less than $5 \%$ are found in early-onset Alzheimer's patients with autosomal dominant inheritance [4].

In our study, EOAD causing gene mutation rate, PSEN1 gene mutation frequency and PSEN2 gene mutation frequency are found $13.58 \%$ (11 out of 81 patients), $63 \%$ ( 7 out of 11 patients) and $36 \%$ (4 out of 11 patients), respectively. EOAD causing gene mutation rate and PSEN1 gene mutation frequency are consistent with the literature. To our knowledge, APP mutations are more common than PSEN2 gene mutations. However, we did not find any mutations in APP gene. These results suggested that APP gene mutations may not be common in Turkish patients as expected. When we compared the age of onset of the patients, age of onset is younger in patients with PSENs mutations as expected, and there was no significant difference between the patients with PSEN1 and PSEN2 mutations (p: 0.06). In our patients with PSEN1 mutations, the earliest onset age was 34 and the latest age was 61; while the earliest onset age was 52 and the latest age was 54 in PSEN2 group. These findings are not surprising because the age of onset shows variability in PSEN1 and PSEN2 mutations.

We also found two variations not previously described in the Alzheimer mutation databases. One of these changes is the V193G variant in the PSEN1 gene and the other one is c.142-7delC variant in the PSEN2 gene. The V193G variation has not been reported in www.molgen.vib-ua.be/ADMutations and www. alzforum.org databases. The V191A variation, located in a position very close to the codon 193 , is characterized as benign in www.molgen.vib-ua.be/ADMutations database. In exac.broadinstitute.org database, the V193G variant was assessed as pathogenic and the allele frequency was specified as "singleton". The V191A variation was also reported as benign in this database and the allele frequency was "1/10000", that means that the V193G variation is less common than the V191A variation. Therefore, we suggested that V193G variant may be pathogenic. PSEN2 c.142-7delC variation is in intron 4-5 and not defined yet in the literature. Mutation was analyzed by Mutation Taster program which predicted the pathogenic nature. In addition, this change may have a disruptive effect on protein function because its localization is nearby the splice site. Family segregation and further analysis needed to make a clear interpretation for these two variants.

In the literature, Lohmann and colleagues [5] analyzed 106 demented Turkish patients from 98 families and detected L134R, H163R, H214E, P264L, and A396T mutations in PSEN1 and R62H, M174V, and S130L changes in PSEN2 gene. We compared the patients with our patients who carry $\mathrm{H} 163 \mathrm{R}$ and $\mathrm{R} 62 \mathrm{H}$ variations. PSEN1 H163R mutated patient had an onset age of 41 years and the initial symptom was forgetfulness and the patient became unable to take care of himself within two years. Visual hallucinations, rigidity, bradykinesia developed after one year and the patient completely stopped talking within a few months. He became completely dependent on the bed when he was 49 . This patient's disease onset age is very close to our patient. PSEN2 R62H patient's memory problems started when he was 63-years-old and the MMSE score was 22 when he was 70 . The patient also had a hemicolectomy story due to colon cancer when he was 55 [5]. This patient 
has dementia and cancer like our patient. Previously, To and colleagues [6] detected PSEN2 R62H and PSEN2 $\mathrm{R} 71 \mathrm{~W}$ variants in several patients diagnosed as breast cancer and reported that these PSEN2 variants may predispose to cancer development. As we know from previous studies, PSEN2 N141I variant increases the cancer risk by inhibiting NOTCH-mediated apoptosis, but there is not efficient data in the literature regarding association between cancer risk and PSEN2 R62H and $\mathrm{R} 71 \mathrm{~W}$ variants [7].

In our country, there are two studies including APOE gene polymorphisms and lipid profile in Alzheimer's disease and PSEN1 and PSEN2 mutation frequencies in Turkish dementia patients $[5,8]$. This is the first study to evaluate age onset and determine mutational frequency of EOAD in Turkish EOAD patients. The PSEN1 mutation rate in our study group was $63 \%$ (7 of 11 patients), which is consistent with the literature. PSEN2 mutations are not common in the literature and APP mutations have higher frequency than PSEN2 mutations, but the PSEN2 mutation rate in our patient group was $36 \%$ (4 out of 11 patients) and any APP mutations were detected. This finding can be explained by the small number of patients or APP gene mutations are not frequent in our population. Further studies with more patients are required to exclude this possibility. To our knowledge, duplications in APP gene can cause Alzheimer's disease and are less frequent in dementia patients. The frequency of mutations may vary depending on ethnicity, therefore further analysis is needed to determine APP duplications frequency in Turkish population. Knowing the mutation frequencies is necessary to determine the diagnostic genetic testing strategy and to provide more appropriate genetic counseling to families. Therefore, it is very important that populations determine their own mutation frequencies.

\section{References}

1. Goldman JS, Hahn SE, Catania JW, LaRusse-Eckert S, Butson MB, et al. (2011) Genetic counseling and testing for alzheimer disease: Joint practice guidelines of the American College of Medical Genetics and the National Society of Genetic Counselors. Genet Med 13: 597-605.

2. Cummings $\mathrm{J}$ (2012) Alzheimer's disease diagnostic criteria: Practical applications. Alzheimers Res Ther 4: 35.

3. Caselli RJ, Beach TG, Knopman DS, Graff-Radford NR (2017) Alzheimer disease: Scientific breakthroughs and translational challenges. Mayo Clin Proc 92: 978-994.

4. Cruts M, Theuns J, Van Broeckhoven C (2012) Locusspesific mutation databases for neurodegenerative brain diseases. Hum Mutat 33: 1340-1344.

5. Lohmann E, Guerreiro RJ, Erginel-Unaltuna N, Gurunlian $\mathrm{N}$, Bilgic $\mathrm{B}$, et al. (2012) Identification of PSEN1 and PSEN2 gene mutations and variants in Turkish dementia patients. Neurobiol Aging 33: 1850.e17-1850.e27.

6. To MD, Gokgoz N, Doyle TG, Donoviel DB, Knight JA, et al. (2006) Functional characterization of novel presenilin-2 variants identified in human breast cancers. Oncogene 25 : 3557-3564.

7. Da Costa CA, Paitel E, Mattson MP, Amson R, Telerman A, et al. (2002) Wild-type and mutated presenilins 2 trigger p53-dependent apoptosis and down-regulate presenilin 1 expression in HEK293 human cells and in murine neurons. Proc Natl Acad Sci USA 99: 4043-4048.

8. Ağaçhan B (1997) Demansiyal hastalıklarda "Özellikle Alzheimer hastalığında" apolipoprotein E gen poliforfizminin lipid profllleri ile olan ilişkisi/Relationship between aplipoprotein $\mathrm{E}$ gene polymorphism and lipid profiles in dementia especially in alzheimer's disease. İstanbul Üniversitesi, İstanbul, Türkiye. 